\title{
Photoelectric effects and magnetic responses in highly rectifying $\mathrm{Nd}_{0.7} \mathrm{Sr}_{0.3} \mathrm{MnO}_{3} / \mathrm{Nb}-\mathrm{SrTiO}_{3}$ heterojunctions
}

\author{
J. F. Wang and J. Gao a) \\ Department of Physics, The University of Hong Kong, Pokfulam Road, Hong Kong
}

(Presented 15 November 2010; received 23 September 2010; accepted 5 November 2010; published online 21 March 2011)

\begin{abstract}
Heterostructures composed of $\mathrm{Nd}_{0.7} \mathrm{Sr}_{0.3} \mathrm{MnO}_{3}$ and $\mathrm{Nb}-\mathrm{SrTiO}_{3}$ were fabricated by laser ablation and characterized under different fields (optic, electric, and magnetic). The asymmetric ratios $[\beta=I(1 \mathrm{~V}) / I(-1 \mathrm{~V})]$ in $I-V$ curves were over $10^{7}$, demonstrating highly rectifying properties in a wide temperature range. Under magnetic fields, remarkable modulations of $I-V$ curves were achieved. Significant open circuit voltages were observed when illuminated by visible lights with wavelengths of $532 \mathrm{~nm}$ and $650 \mathrm{~nm}$. A planar back-to-back diode $\left(\mathrm{Nd}_{0.7} \mathrm{Sr}_{0.3} \mathrm{MnO}_{3} / \mathrm{Nb}-\mathrm{SrTiO}_{3} /\right.$ $\mathrm{Nd}_{0.7} \mathrm{Sr}_{0.3} \mathrm{MnO}_{3}$ ) with a good on/off ratio to visible lights was also fabricated. These results were discussed in analogy with conventional semiconductor junctions. (C) 2011 American Institute of Physics. [doi:10.1063/1.3540675]
\end{abstract}

\section{INTRODUCTION}

Recently, there have been intensive studies on heterostructures composed of complex oxides. ${ }^{1,2}$ It has been expected that their properties could be more striking than those found in semiconductor heterostructures. Rectifying properties, bias tunable magnetoresistance, and photoelectric effects have been reported in perovskite-oxide heterojunctions composed of manganites and titanates. ${ }^{3-11}$ Colossal magnetoresistance manganites are typical complex oxides with strongly correlated interactions. Strontium titanate $\left(\mathrm{SrTiO}_{3}\right)$ is a wide-band-gap semiconductor and has good lattice match with manganites. When doped with $\mathrm{Nb}$ or $\mathrm{La}$, $\mathrm{SrTiO}_{3}$ becomes n-type conducting. Simply depositing a manganite film on an $\mathrm{Nb}$ doped (or La doped) $\mathrm{SrTiO}_{3}$ substrate can form a single-interface junction. ${ }^{4}$ In general, these properties should strongly depend on the barrier profiles at the interface. Although the detailed descriptions of barrier profiles are hindered by the strong correlations, it is enlightening to analyze them using conventional semiconductor theories. Within such theories, energy barriers are formed at the interfaces due to the difference of Fermi levels in the materials constituting the junction. ${ }^{12}$ Based on the analysis of the $I-V$ and $C-V$ characteristics, Sawa et al. concluded that the energy barriers in $\mathrm{Pr}_{0.7} \mathrm{Ca}_{03} \mathrm{MnO}_{3} / \mathrm{Nb}-\mathrm{SrTiO}_{3}$ and $\mathrm{La}_{0.7} \mathrm{Sr}_{0.3} \mathrm{MnO}_{3} / \mathrm{Nb}-\mathrm{SrTiO}_{3}$ junctions are $\sim 0.7$ and $\sim 0.6 \mathrm{eV}$, respectively. ${ }^{8}$ Susaki et al. found that the $I-V$ curves of $\mathrm{La}_{0.7} \mathrm{Sr}_{0.3} \mathrm{MnO}_{3-\delta} / \mathrm{Nb}-\mathrm{SrTiO}_{3}$ junctions can be well described by thermally assisted tunneling with an effectively temperature-independent Schottky barrier, which is $\sim 0.86 \mathrm{eV}$ for the oxygen deficient junction and $\sim 0.66 \mathrm{eV}$ for stoichiometric one. ${ }^{10}$ An even higher barrier height of $\sim 1.64 \mathrm{eV}$ was deduced for $\mathrm{La}_{0.7} \mathrm{Ca}_{0.3} \mathrm{MnO}_{3} / \mathrm{Nb}-\mathrm{SrTiO}_{3}$ junctions by using Fowler theory to fit photoelectric experimental results. ${ }^{11}$ Despite such values of barrier height, the reported open

\footnotetext{
a) Author to whom correspondence should be addressed. Electronic mail: jugao@hku.hk.
}

circuit voltages $V_{O C}$ are quite small. ${ }^{6,9,11}$ In this paper, we present a study on photoelectric effects and magnetic/electric responses for highly rectifying heterojunctions composed of $\mathrm{Nd}_{0.7} \mathrm{Sr}_{0.3} \mathrm{MnO}_{3}$ (NSMO) and 0.05 wt. \% Nb-doped $\mathrm{SrTiO}_{3}$ (Nb-STO). Remarkable magnetic field modulations are observed and pronounced photovoltages are achieved.

\section{EXPERIMENTAL PROCEDURES}

Epitaxial NSMO thin films (100 nm) were deposited on Nb-STO (001) substrates by pulsed laser deposition at 720 ${ }^{\circ} \mathrm{C}$ in $1 \mathrm{mbar}_{2}$. The grown samples were annealed at grown temperature in oxygen of 0.5 bar for half an hour and then slowly cooled down to room temperature. As revealed by xray spectra, these films are of single phase and have good epitaxy. Conventional photolithography technique was used to pattern the films into areas of $1 \times 1 \mathrm{~mm}^{2}$. Silver $(\mathrm{Ag})$ pads were evaporated onto the NSMO films and Nb-STO substrates as electrodes. The schematic view of the junctions is displayed in the inset of Fig. 1(b). Current voltage characteristics were measured using two-lead-probe method and the positive voltage applied on NSMO was defined as the forward bias. For MR measurements, the magnetic fields were applied perpendicular to the film plane. For photoelectric experiments, semiconductor laser diodes with wavelengths of 532 and $650 \mathrm{~nm}$ were used as light sources.

\section{RESULTS AND DISCUSSIONS}

Figure 1(a) presents the current voltage curves of a junction measured at different temperatures. The asymmetric ratios, $\beta=I(1 \mathrm{~V}) / I(-1 \mathrm{~V})$, are over $10^{7}$, demonstrating highly rectifying properties. Hysteresis is observed under negative bias voltages, but is absent under positive ones. ${ }^{8}$ A magnified view for the forward direction indicates that $\log J-V$ curves are essentially straight under small bias voltages and the linearity is deviated under larger bias voltages.

To further understand these junctions, detailed analyses are performed using both Schottky junction and p-n junction 

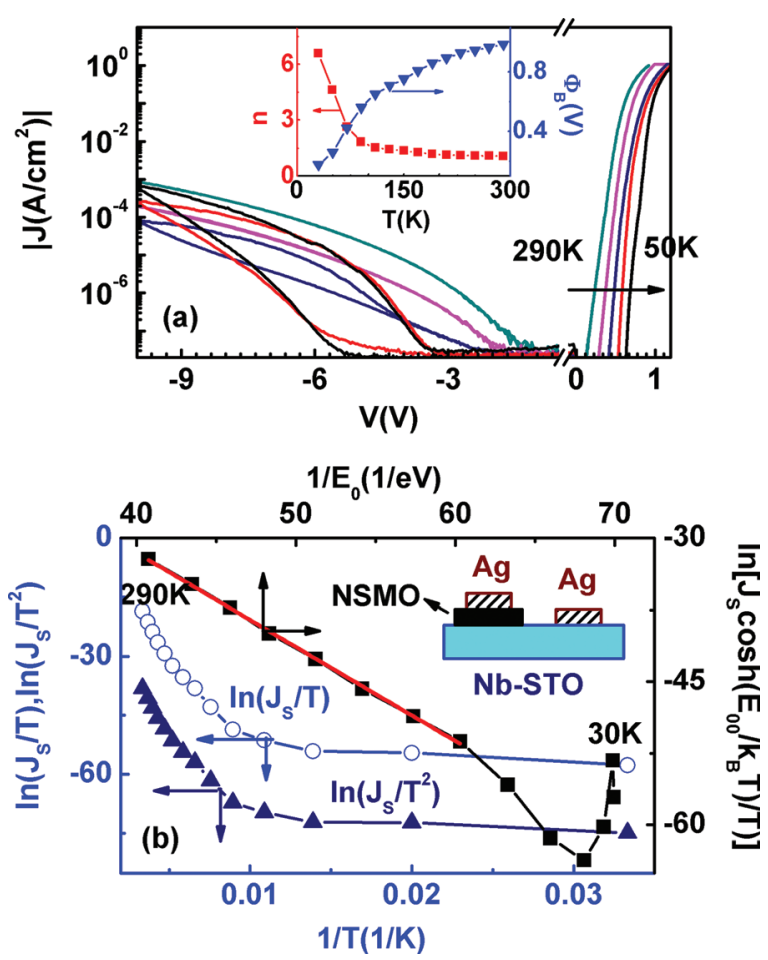

FIG. 1. (Color online) (a) $\log |J|-V$ curves measured at 290, 230, 170, 110, and $50 \mathrm{~K}$, respectively. The inset: temperature dependences of ideality factor $n$ and barrier height $\Phi_{B}$; (b) $\ln \left(J_{S} / T\right)-1 / T, \ln \left(J_{S} / T^{2}\right)-1 / T$, and $\ln \left[J_{S} \cosh \left(E_{00} / k_{B} T\right) /\right.$ $T]-1 / E_{0}$ characteristics for the temperature range 30-290 K. The inset: schematic view of the junction.

models. For either kind of junction, the $J-V$ relation in the forward direction can be expressed as $J \approx J_{S} \exp \left(q V / n k_{B} T\right)$ when $q V \gg k_{B} T$, where $J_{S}$ is the saturation current density, $n$ the ideality factor, and $k_{B}$ the Boltzmann constant. ${ }^{12}$ For a perfect $\mathrm{p}-\mathrm{n}$ junction or a Schottky junction with purely thermal emission process, $n$ is 1 . In the studied junction, $n$ at room temperature is evaluated to be 1.1 , very close to 1 . This may reflect the applicability of such models. One way to deduce the energy barrier is to get the slopes of $\ln \left(J_{S} / T\right)-1 /$ $T$ plot for a p-n junction and $\ln \left(J_{S} / T^{2}\right)-1 / T$ plot for a Schottky junction, ${ }^{8}$ as displayed in Fig. 1(b). These plots are almost linear above $200 \mathrm{~K}$ and the slopes give the energy barriers of $\sim 0.7 \mathrm{eV}$. For a Schottky diode in a pure thermionic process case, $J_{S}$ can be expressed as $J_{S}=A^{*} T^{2} \exp \left(-q \Phi_{B} / k_{B} T\right)$, in which $A^{*}$ is effective Richardson constant and $q \Phi_{B}$ the Schottky barrier height. By setting $A^{*}$ to be $156 \mathrm{~A} / \mathrm{cm}^{2} \mathrm{~K}^{2}$ for $\mathrm{Nb}-\mathrm{STO}, q \Phi_{B}$ can be estimated, as given in the inset of Fig. 1(a). At room temperature, $q \Phi_{B}$ is $\sim 1 \mathrm{eV}$, much higher than those deduced using the first method. With decreasing temperature, it decreases and is reduced to $\sim 0.2 \mathrm{eV}$ when the temperature is lowered to 30 $\mathrm{K}$. At the same time, $n$ is increased from $\sim 1.1$ at $290 \mathrm{~K}$ to $\sim 6.6$ at $30 \mathrm{~K}$. These anomalous behaviors at low temperatures are probably because the low-temperature transport is direct tunneling process $\left[J=J_{S} \exp \left(q V / E_{00}\right)\right]^{10,12}$ Correspondingly, at intermediate temperatures, it should be thermal assisted tunneling $\left[J=J_{S} \exp \left(q V / E_{0}\right), E_{0}=E_{00} \operatorname{coth}\left(E_{00} /\right.\right.$ $\left.\left.k_{B} T\right)\right]$. For such a process, the slope of $\ln \left[J_{S} \cosh \left(E_{00} / k_{B} T\right) / T\right]$ vs $1 / E_{0}$ gives $-q \Phi_{B}$. As shown in Fig. 1(b), this plot essentially follows a straight line above $150 \mathrm{~K}$ and the determined

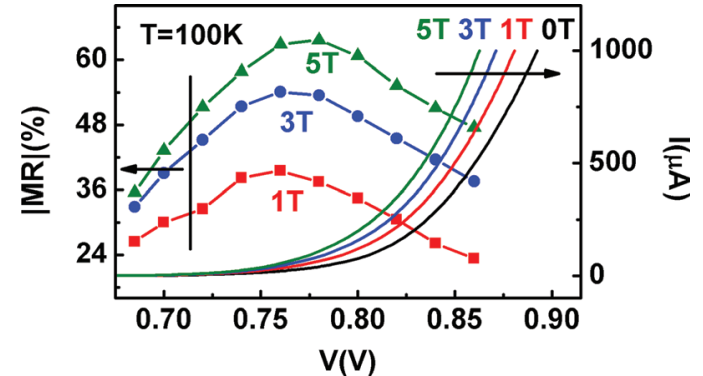

FIG. 2. (Color online) $I-V$ curves and $|M R|$ in the forward direction at 100 $\mathrm{K}$ under magnetic fields of $0,1,3$, and $5 \mathrm{~T}$.

barrier height is $\sim 1 \mathrm{eV}$, which is the same as that at room temperature estimated using the effective Richardson constant.

Distinct magnetic field effects were observed in these junctions. Figure 2 depicts the $I-V$ curves in the forward direction recorded at $100 \mathrm{~K}$ under magnetic fields of $0,1,3$, and 5 T. Clearly, magetoresistance $M R$, defined as $M R(V, H)=100 \% \times[R(V, H)-R(V, 0)] / R(V, 0)$, is bias dependent. With a fixed bias voltage $V$, current $I$ increases with increasing magnetic fields, indicating negative $M R$. The maximum magnitudes of $M R$ are $\sim 40 \%, \sim 54 \%$, and $\sim 64 \%$ for the magnetic fields of 1,3 , and $5 \mathrm{~T}$, respectively.

As shown in Fig. 3(a), the $I-V$ curves have clear responses to visible lights. When the junction is illuminated, carriers will be excited by incident photons, producing the photocurrents. Naively, current under illumination $I_{\text {light }}$ is simply the superposition of dark current $I_{\text {dark }}$ and photocurrent $I_{L}, I_{\text {light }}=I_{\text {dark }}+I_{L}$. As can be seen from Fig. 3(a), the calculated and measured light $I-V$ curves are qualitatively same. The differences at higher positive bias voltages are understandable since the barrier height would be lowered
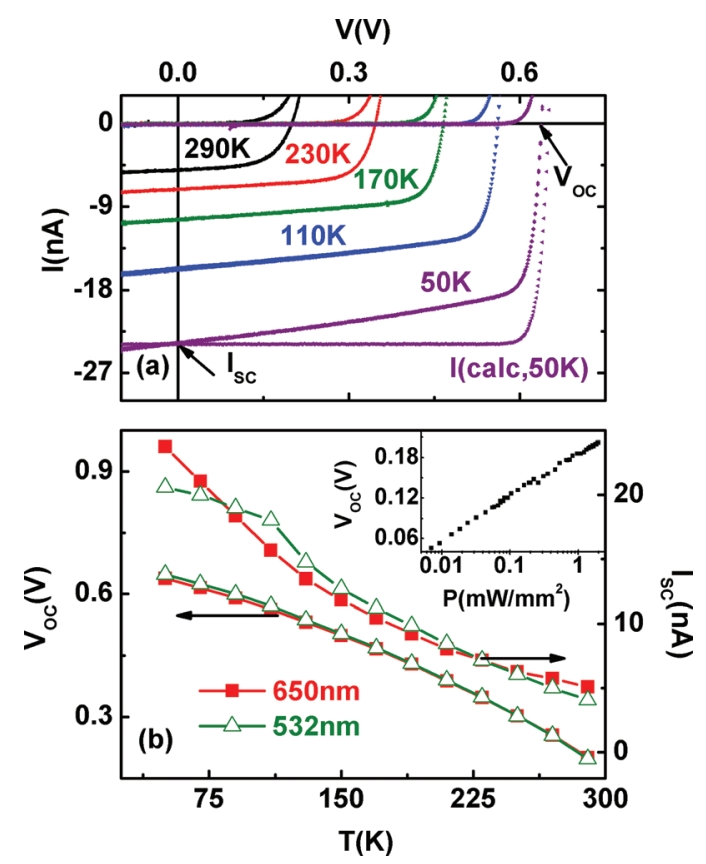

FIG. 3. (Color online) (a) $I-V$ curves with and without light $(\lambda=650 \mathrm{~nm}$, $P=2 \mathrm{~mW} / \mathrm{mm}^{2}$ ) at different temperatures and a calculated light $I-V$ curve at $50 \mathrm{~K}$; (b) temperature dependences of $V_{O C}$ and $I_{s c}(\lambda=650 \mathrm{~nm}$ and $532 \mathrm{~nm}$, $\left.P=2 \mathrm{~mW} / \mathrm{mm}^{2}\right)$. The inset: $V_{O C}-\log P$ plot at $290 \mathrm{~K}(\lambda=532 \mathrm{~nm})$. 


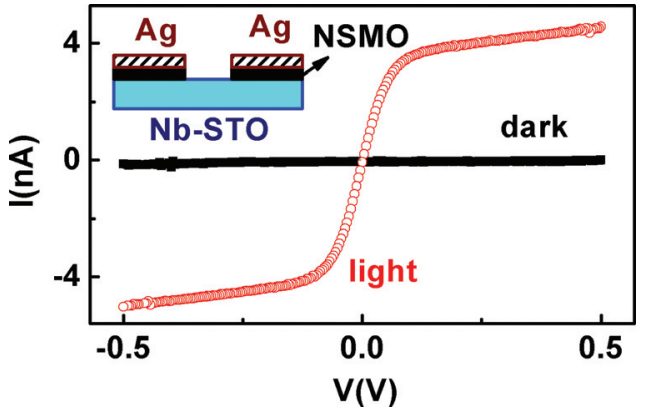

FIG. 4. (Color online) Photoresponse $(\lambda=650 \mathrm{~nm})$ of a NSMO/Nb-STO/ NSMO diode at $290 \mathrm{~K}$. The inset: schematic diagram of the device.

under a forward bias. One of the important parameters in photoelectric measurements is the open circuit voltage $V_{O C}$, which occurs when the current in the external circuit equals zero. The temperature dependences of $V_{O C}$ are presented in Fig. 3(b). $V_{O C}$ is $\sim 0.2 \mathrm{~V}$ at room temperature. When the temperature is lowered to $50 \mathrm{~K}$, it increases to $\sim 0.7 \mathrm{~V}$. This value is comparable to the barrier heights deduced in a previous section. Similar to $V_{O C}$, short circuit current $I_{S C}$ increases as the temperature decreases. It is of interest to study the dependences of $V_{O C}$ and $I_{S C}$ on light density $P$. It is found that a linear dependence exists between the short circuit current $I_{S C}$ and light density $P$. This is reasonable since $I_{S C}$ should be proportional to $\eta P$, where $\eta$ is the conversion efficiency. Different from $I_{S C}-P$ plot, $V_{O C}$ is proportional to $\ln P$, as displayed in the inset of Fig. 3(b). Such a dependence is the same as that in conventional p-n junctions and Schottky junctions, where $V_{O C} \sim k_{B} T \ln \left(I_{L} / \mathrm{I}_{\mathrm{S}}\right) / q .{ }^{12}$

To explore potential applications of such heterojunctions, a planar back-to-back diode (NSMO/Nb-STO/NSMO) was fabricated, as illustrated in the inset of Fig. 4. This piece of prototype device exhibits a good on/off ratio (over 200 at $0.5 \mathrm{~V}$ ) to light illumination. The performances under positive and negative biases are essentially symmetric. With light illumination, the photocurrent first rises with increasing voltage and then shows a saturated feature. The increase of photocurrent at low bias is probably due to the expansion of barrier region.

\section{CONCLUSIONS}

$\mathrm{NSMO} / \mathrm{Nb}-\mathrm{STO}$ heterojunctions fabricated by pulsed laser deposition demonstrate excellent rectification properties, bias tunable magnetoresistance, and strong photoelectric effects. In a wide temperature range, the asymmetric ratios $[\beta=I(1 \mathrm{~V}) / I(-1 \mathrm{~V})]$, are over $10^{7}$. In a magnetic field of $5 \mathrm{~T}$, the maximum $M R$ at $100 \mathrm{~K}$ is $\sim-64 \%$. The open circuit voltage is $\sim 0.2 \mathrm{~V}$ at room temperature and increases to $\sim 0.7$ $\mathrm{V}$ when the temperature is lowered to $50 \mathrm{~K}$. A prototype planar back-to-back diode (NSMO/Nb-STO/NSMO) with a good on/off ratio (over 200) to visible lights has also been fabricated. Conventional semiconductor theories are used to discuss these results.

\section{ACKNOWLEDGMENTS}

This work has been supported by a grant of the Research Grant Council of Hong Kong (Project No. HKU 7024/07P) and a seed funding of the University of Hong Kong.

${ }^{1}$ J. Mannhart and D. G. Schlom, Science 327,1607 (2010).

${ }^{2}$ H. Takagi and H. Y. Hwang, Science 327, 1601 (2010).

${ }^{3}$ M. Sugiura, K. Uragou, M. Noda, M. Tachiki, and T. Kobayashi, Jap. J. Appl. Phys. 38, 2675 (1999).

${ }^{4}$ H. Tanaka, J. Zhang, and T. Kawai, Phys. Rev. Lett. 88, 027204 (2001).

${ }^{5}$ F. M. Postma, R. Ramaneti, T. Banerjee, H. Gokcan, E. Haq, D. H. A. Blank, R. Jansen, and J. C. Lodder, J. Appl. Phys. 95, 7324 (2004).

${ }^{6}$ J. R. Sun, B. G. Shen, Z. G. Sheng, and Y. P. Sun, Appl. Phys. Lett. 85, 3375 (2004)

${ }^{7}$ N. Nakagawa, M. Asai, Y. Mukunoki, T. Susaki, and H. Y. Hwang, Appl. Phys. Lett. 86, 082504. (2005).

${ }^{8}$ A. Sawa, T. Fujii, M. Kawasaki, and Y. Tokura, Appl. Phys. Lett. 86, 112508 (2005).

${ }^{9}$ Z. Luo and J. Gao, J. Appl. Phys. 100, 056104 (2006).

${ }^{10}$ T. Susaki, N. Nakagawa, and H. Y. Hwang, Phys. Rev. B 75, 104409 (2007).

${ }^{11}$ Z. Luo, J. Gao, A. B. Djurisic, C. T. Yip, and G. B. Zhang, Appl. Phys. Lett. 92, 182501 (2008).

${ }^{12}$ S. M. Sze and K. K. Ng, Physics of Semiconductor Devices, 3rd ed. (John Wiley \& Sons, Inc., Hoboken, New Jersey, 2006). 